\title{
Contrasting Confocal with Defocusing Micro-Scale Spatially Offset Raman Spectroscopy
}

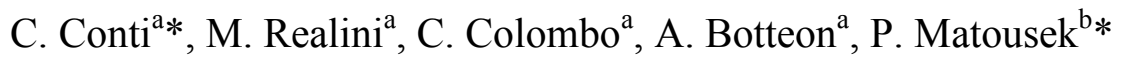

${ }^{a}$ Consiglio Nazionale delle Ricerche, Istituto per la Conservazione e la Valorizzazione dei Beni Culturali (ICVBC), Via Cozzi 53, 20125, Milano, Italy.

${ }^{b}$ Central Laser Facility, Research Complex at Harwell, STFC Rutherford Appleton Laboratory, Harwell Oxford, OX110QX, United Kingdom.

* Corresponding authors: conti@icvbc.cnr.it ; pavel.matousek@stfc.ac.uk

Key words: Spatially offset Raman spectroscopy, subsurface, non-destructive, diffusely scattering, Cultural Heritage

\begin{abstract}
The study compares and contrasts conventional confocal Raman microscopy/spectroscopy (CRM) with a recently developed micrometer scale defocusing Spatially Offset Raman Spectroscopy (micro-SORS), a method providing a new analytical capability for investigating non-destructively the chemical composition of subsurface, micrometer-scale-thick diffusely scattering layers at depths beyond the reach of CRM. Due to close similarities between the two techniques and comparable embodiment of the instrumentations, but radically different interpretations of data, it is crucially important to recognise which type of method is pertinent to
\end{abstract}


a specific measurement. The distinction comes principally from the nature of sample, whether turbid (micro-SORS measurement) or transparent (CRM measurement) on the spatial scale of the axial (z-)scan of the measurement. Which type of sample one deals with may not always be easily recognisable with micro-scale thick layers and the study therefore also presents a simple method for suggesting whether CRM or micro-SORS methodology applies. This test relies on an axial (z-)scan performed through the sample in both the positive and negative directions from the normal, imaged sample surface position using conventional CRM instrument. The absence or presence of symmetry or asymmetry of the intensity profiles of measured Raman signals around the imaged sample surface position as a function of sample axial displacement then suggests which interpretation could apply. The study paves a way for the development of micro-SORS as a widely applicable analytical tool deployable on conventional Raman microscopes.

\section{Introduction}

A recently developed technique of defocusing micro-Spatially Offset Raman Spectroscopy (micro-SORS) provides a new analytical capability for interrogating non-invasively the chemical makeup of thin stratified layers in highly diffusely scattering (turbid) samples ${ }^{1,2,3}$. Such layers can be found, for example, in Cultural Heritage with paints (painted statues, mural paintings or on other decorated objects) ${ }^{4}$. Highly turbid layers are also common in other areas including biological, forensic, polymer and paper samples ${ }^{5}$.

The defocusing micro-SORS derives its properties from its parent technique Spatially Offset Raman Spectroscopy (SORS) ${ }^{6,7}$. In essence it combines SORS with microscope imaging concepts. Its key principles have been described in detail earlier ${ }^{1,4,5}$. In brief, defocusing micro- 
SORS relies on collecting at least two Raman spectra using a Raman microscope; the first one, with the sample in a conventional 'imaged' position, where surface of the sample is sharply imaged, and the second, obtained after repositioning the sample, typically, away from the microscope objective by a 'defocusing distance $\Delta \mathrm{z}$ ', in a 'defocused' position (or by repositioning the microscope objective away from sample surface where microscope objective is mounted a motorised stage). The sample displacement $\Delta \mathrm{z}$ ('z-scan') leads to the defocusing of both the laser illumination and Raman collection zones on sample surface and their consequential enlargements (see Figure 1). The concept of scaled subtraction was first applied to confocal Raman microscopy with transparent samples by the Morris group ${ }^{8,9}$.

The classical SORS spatial offset ${ }^{6}$ is present in this arrangement only at the level of individual photons; each detected Raman photon can be tracked back to its originating laser photon. With enlarged illumination and collection zones, the exit point of a detected Raman photon can be spatially separated at sample surface from its originating laser photon when entering sample. This photon-pair spatial offset can range from no separation to a certain maximum value given by the diameter of the laser illumination/Raman collection zone. The entire ensemble of Raman photons detected in this way will therefore have a certain distribution of spatial offsets from their originating laser photons. As such there will be a non-zero net SORS spatial offset present in the overall Raman spectrum acquired at the 'defocused' position. The magnitude of this net spatial offset can be controlled by the overall degree of defocusing introduced, ie the diameter of the illumination/Raman collection zone - the larger defocusing leading to the larger effective net spatial offset. In turn, the 'imaged' collection yields a near zero-spatially offset Raman spectrum due to their deposition and collection zones being confined to effectively a single spot. 
As such the 'imaged' measurement yields a Raman spectrum typically dominated by the surface layer and corresponds conceptually to a zero-spatially offset measurement in conventional SORS analysis. In contrast, the 'defocused' measurement yields a Raman spectrum which has a higher degree of Raman signal contributions from sublayers. For a two-layer system, a simple numerical processing involving a scaled subtraction of the 'imaged' spectrum from the 'defocused' spectrum aiming at cancelling the contributions from the top layer can be used to retrieve the pure Raman spectrum of sublayer. The pure Raman spectrum of the top layer can be obtained in analogy in reverse when a contribution of the sublayer occurs in the 'imaged' measurement.

In contrast, in the CRM measurements, the depth information is obtained by a radically different mechanism - by moving the laser beam and Raman collection zones through the inner compartment of the (transparent) sample (z-scan) and collecting primarily Raman signal from the imaged zone within the sample and as such providing direct information on the internal composition of the sample as a function of z-scan (see Figure 2). This should be contrasted with defocusing micro-SORS where the depth access is facilitated by a 'z-scan' resulting only in altering the diameters of the illumination and collection zones on sample surface with subsequent photon diffusion into the sample with no formation of any equivalent laser/image 'focal zone' within the sample.

As the same instrumentation is employed in both the types of measurements a potentially confusing situation can arise in cases where the nature of sample, ie whether transparent or 
turbid, is not known a priory and as such a wrong interpretation to data could be applied. As mentioned above the distinction between the two methods is governed by the turbidity of sample alone; when the sample is transparent on the scale of the z-scan (z-scan magnitude >> photon transport path length in the sample) $)^{2}$ the CRM interpretation of data is relevant and when the sample is diffusely scattering on the scale of the z-scan the micro-SORS interpretation applies. As in some situations it may be difficult to determine whether the sample belongs to one category or the other the following simple test can be performed to suggest whether the measurement belongs to CRM or micro-SORS category. The proposed methodology relies on performing a simple axial (z-)scan in both the positive and negative directions starting from the 'imaged' position with a conventional Raman microscope (see Figure 3).

In a 'CRM measurement', that is with a transparent sample, the movement of the laser beam and image zone into the object will lead to a largely sustained Raman signal levels for homogeneous samples, or reflecting the internal sample constitution which is directly imaged for inhomogeneous samples. However moving the laser beam and collection zone above the transparent sample leads to a diminishment of detected Raman signals (the residual signal is principally caused by Raman signal sensed through the emission of Raman photons directly into the collection cone ${ }^{10}$ ).

In contrast, in 'micro-SORS measurement', that is with a turbid sample on the scale of the ' $\mathrm{z}$ scan' measurement, the movement of the laser beam and imaged zone below or above the sample surface will produce a very similar Raman response as a similar degree of laser illumination and Raman collection zone enlargement on sample surface is induced. As such a symmetric response 
would be present around the origin ('imaged' position) in a 'z-scan' for any detected layer. From the symmetry/asymmetry around the origin obtained through such $+/$ - z-scan one could therefore start recognising which type of data one deals with, ie whether of micro-SORS or CRM nature. The presence of asymmetry within any of the detected signals suggests the presence of a transparent sample (CRM measurement). However, the presence of symmetry does not prove one deals definitely with a turbid sample (micro-SORS measurement) as a symmetric response could also be obtained from a thin transparent sample if its thickness was equal or lower than the z- (depth) resolution of the Raman system. In the intermediate thickness range, if a transparent sample was gradually becoming thinner, starting from a semi-infinite thickness, one would see a gradual decrease of asymmetry reaching full symmetry when sample reached the thickness comparable to the z-resolution of the optical system of the Raman instrument. An example of a thin transparent sample which could produce a symmetric response with high likelihood is a thin ( $1 \mu \mathrm{m}$ thick) polymeric film with no extended substrate below it. A caution when interpreting the data therefore needs to be exercised.

As the micro-SORS measurement is traditionally performed by moving the sample away from the microscope objective the data is less likely to be confused with CRM interpretation as transparent samples would be expected to produce lower level Raman signals in this z-scan region (stemming from the abovementioned residual Raman signal sensed due to emission of Raman photons into the collection cone). However, when performing supposedly a CRM measurement on a highly turbid sample (on the scale of the z-scans performed) an incorrect interpretation would be used if the sample was thought of being transparent as the measured Raman z-dependencies would be interpreted as due to the laser beam and Raman collection 
movement through the sample with the z-scan, ie not due to photon diffusion into the sample, an unrelated process.

It should be noted that the proposed test is only valid in situations where sample can be considered semi-infinite in depth on the scale of the measured depths and where it does not exhibit significant absorption on the same scale. (It is noted that if the top layer was thin it could also produce a symmetric response irrespective of whether turbid or transparent.) In multilayer cases, it can also only be applied in situations where all the layers are of the same optical nature, ie are either turbid or transparent (eg multiple paint layers in art restoration).

The simple test is also not applicable with transparent samples in situations where the surface or layer interfaces are not optically flat. This is due to the fact that imperfect optical surface (eg rough surface) may preclude moving the laser focal spot and the Raman collection zone into the depth of the sample overlapped and tightly imaged and may lead to a gradual loss of imaging from deeper within the sample, especially when measuring with confocal aperture, which makes Raman microscopy particularly sensitive to interface imperfection. Such an effect could also confuse the interpretation of measured dependencies.

\section{Experimental}

\section{Specimens}

For transparent measurements two polymeric layers were used, polystyrene (PS) and polyethylene terephthalate (PET) (Table 1). The first measurement was performed using a single polystyrene (PS) sheet. The second measurement was carried out on a two-layer sheet of 
polystyrene (PS) as the bottom layer covered with the polyethylene terephthalate (PET) top layer.

For diffusely scattering samples two painted layer were used, phthalocyanine blue and red ochre (Table 1). The first measurement was performed using a single layer of phthalocyanine blue. The second measurement was carried out on a two-layer painted system made of phthalocyanine blue as the bottom layer covered with the red ochre top layer. None of the pigments exhibits significant absorption at the laser excitation wavelength ${ }^{11,12}$.

\section{Raman spectroscopy}

Both the micro-SORS and CRM measurements were carried out under identical experimental conditions using a Senterra dispersive micro-Raman spectrometer (Bruker) with a 1200 grooves/mm grating and coupled to an Olympus BX51 microscope using 20x objective. The laser excitation wavelength was $785 \mathrm{~nm}$ with a power at the sample of $\sim 10 \mathrm{~mW}$. The Raman spectra were acquired using a Peltier cooled CCD detector (1024 x 256 pixels). The measurements were carried out both with and without confocal aperture ('pinhole'). The diameter of the confocal aperture was $50 \mu \mathrm{m}$. It should be noted that the measurement without the confocal aperture is also partially confocal as the width of spectrograph slit $(50 \mu \mathrm{m})$ acts as a confocal aperture in one dimension in its own right (the spectrograph slit height was $1000 \mu \mathrm{m}$ ). The spectra were acquired with $150 \mathrm{~s}$ acquisition time (5 accumulations, $30 \mathrm{~s}$ each). 
The Raman band intensities were retrieved using OPUS 7.5 (Bruker) performing the K-method integration of one the most intense band for each compound. The pre-processing step consisted of truncating the spectra to $200-1500 \mathrm{~cm}^{-1}$.

\section{Results and Discussion}

First a single-layer samples, turbid (a single layer of paint) and transparent (a polymer sheet) were analysed by the Raman microscope in the proposed +/- z-scan to demonstrate the key difference in the intensity dependencies in micro-SORS (turbid sample) and CRM (transparent sample) measurements. The Raman intensities plotted (747 and $1002 \mathrm{~cm}^{-1}$ for the turbid and transparent layers, respectively) as a function of displacement $\Delta \mathrm{z}$ in individual z scans are shown in Figure 4, where the measurements with and without 'pinhole' are both shown. The measurement performed on the turbid sample shows clear symmetry of Raman intensities around its origin $(\Delta \mathrm{z}=0)$ as expected whereas the measurement on transparent sample using the identical experimental setup and conditions yields strongly asymmetric curve with lower level signal detected when the sample is displaced away from the microscope objective from its 'imaged' surface position. At the negative values of $\Delta z$ with the transparent sample the Raman signal largely persists. The measurements with and without the confocal aperture are qualitatively similar with respect to the presence or absence of symmetry/asymmetry in the two situations.. Overall, the symmetry of the curve indicates the micro-SORS interpretation of data needs to be invoked (unless a very thin transparent samples is present in which case a symmetric response would still be obtained as mentioned above) and the asymmetry indicates the CRM data interpretation applies. 
In a second experiment two-layer samples were analysed; (i) a two-layer sample consisting of transparent polymers and (ii) a two layer turbid sample composed of two layers of paint. Again the intensity of representative Raman bands of individual layers $\left(858 \mathrm{~cm}^{-1}\right.$ for the top layer of transparent two-layer sample and $227 \mathrm{~cm}^{-1}$ for the top layer of turbid two-layer sample) were plotted versus displacement $\Delta z$. The measurements were again performed both with and without 'pinhole'. The results are shown in Figure 5.

The measurement on a turbid two-layer sample produced a symmetric response for all the detected layer signals around the origin $(\Delta \mathrm{z}=0)$ as anticipated due to the fact that the displacement in either direction produces effectively identical enlargement of laser and Raman collection zone on sample surface. This behaviour is indicative of diffusely scattering sample suggesting micro-SORS data interpretation (unless a very thin transparent samples is present in which case a symmetric response would still be obtained as mentioned above). Both the measurements with and without the confocal aperture produced qualitatively similar results.

In contrast, the measurement on the transparent two-layer sample shows an asymmetric behaviour with at least one of the layer signals exhibiting asymmetry, mainly due to signal persistence at negative values of $\Delta \mathrm{z}$ reflecting the internal structure of sample and the lower level of Raman signal at positive values for both the layers. It is noted that the Raman signal of the bottom layer ultimately also diminishes at larger negative values of $\Delta z$, an effect we suggest could be potentially due to imperfect optical surface of the top layer used as this was not observed on the same bulk sublayer when used on its own and therefore could not be due to residual absorption or turbidity within this material (ie without the top layer in place - see Fig. 
4). Nevertheless the asymmetry of the signal around the centre $(\Delta z=0)$ is still clearly evident. The presence of this asymmetry in at least one of the detected layer signals indicates need to invoke CRM data interpretation in this situation.

It is also clear that if one applied the CRM data interpretation to a turbid sample performing zscan incorrect deduction of sample properties would be derived from such measurement as the thickness of any potential stratification derived from the data would be incorrect as the z-scan is unrelated directly to depth but instead to the enlargement of laser and collection zones on sample surface and subsequent photon diffusion processes.

Figure 6 shows typical Raman spectra acquired in the above two layer situations for a measurement with confocal and without confocal aperture. Similar spectra are obtained both with and without the confocal aperture although the ratio between the top and bottom layer and the rate of decay of signals with the $\Delta \mathrm{z}$ can be different in the two situations.

In general, the presence of the confocal aperture does not qualitatively alter the results although its presence can lead to a higher sensitivity to optical quality (flatness) of the layer surfaces as evidenced above. As such it might be advisable to perform the test without the confocal aperture or avoid imperfectly flat optical surfaces altogether.

\section{Conclusions}

The study compares and contrasts conventional CRM with micro-SORS emphasising the radically different nature of their underlying photon propagation mechanisms. A simple test is 
also proposed for indicating which type of interpretation should be applied, ie whether CRM or micro-SORS. The assessment is based around a z-scan carried out in both away and towards the microscope objective from the sample surface 'imaged' position. The dependence of the Raman intensities on the z-scan displacement from the original 'imaged' position and its asymmetry/symmetry the detected layer signals around the origin then suggests on the type of measurement performed; the symmetry in all signals from detected layers suggesting the microSORS process is present and its interpretation should be applied (unless a very thin sample is analysed in which case a transparent sample could still be present) and the asymmetry in any of the signals from detected layers indicating the presence of the CRM process with CRM interpretation applying.

\section{$\underline{\text { Acknowledgement }}$}

We would like to thank Dr. Pezzuto and Dr. Magagnin (Akzo Nobel Coating spa) for their support in the preparation of paint samples.

\section{REFERENCES}

[1] C. Conti, C. Colombo, M. Realini, G. Zerbi, P. Matousek, Appl. Spectrosc. 2014 ; 68, 686.

[2] P. Matousek, C. Conti, C. Colombo, M. Realini, Appl. Spectrosc. 2015 ; 69, 1091.

[3] C. Conti, M. Realini, A. Botteon, C. Colombo, S. Noll, S.R. Elliott, P. Matousek, Appl. Spectrosc., 2015 ; , in press, DOI: 10.1177/0003702815615345.

[4] C. Conti, M. Realini, C. Colombo, P. Matousek, J. Raman Spectrosc. 2015 ; 46, 476.

[5] C. Conti, M. Realini, C. Colombo, K. Sowoidnich, N. Kristian Afseth, M. Bertasa, A. Botteon' P. Matousek, Anal. Chem. 2015 ; 87, 5810. 
[6] P. Matousek, I. P. Clark, E. R. C. Draper, M. D. Morris, A. E. Goodship, N. Everall, M. Towrie, W. F.

Finney, A. W. Parker, Appl. Spectrosc. 2005 ; 59, 393.

[7] K. Buckley, P. Matousek, Analyst 2011 ; 136, 3039.

[8] A. Govil, D.M. Pallister, M.D. Morris, Appl. Spectrosc. 1991; 45, 1604.

[9] A. Govil, D.M. Pallister, M.D. Morris, Appl. Spectrosc. 1993; 47, 75.

[10] N.J. Everall, Appl. Spectrosc. 2009; 63, 245A.

[11] G. Poldi, S. Caglio, Optics Spectrosc., 2013; 114, 929935.

[12] J.L. Mortimore, L.J.R. Marshall, M.J. Almond, P. Hollins, W. Matthews, Spectrochim. Acta Part A, 2004; 60, 11791188.

Table 1: Investigated samples

\begin{tabular}{l|l|l}
\hline \multicolumn{1}{c|}{ Sample } & Type of material & Thickness \\
\hline Polystyrene (PS) & Transparent & $1.5 \mathrm{~mm}$ \\
\hline Polyethylene terephthalate (PET) & Transparent & $100 \mu \mathrm{m}$ \\
\hline Phthalocyanine blue & Turbid & $50 \mu \mathrm{m}$ \\
\hline Red ochre & Turbid & $50 \mu \mathrm{m}$ \\
\hline
\end{tabular}




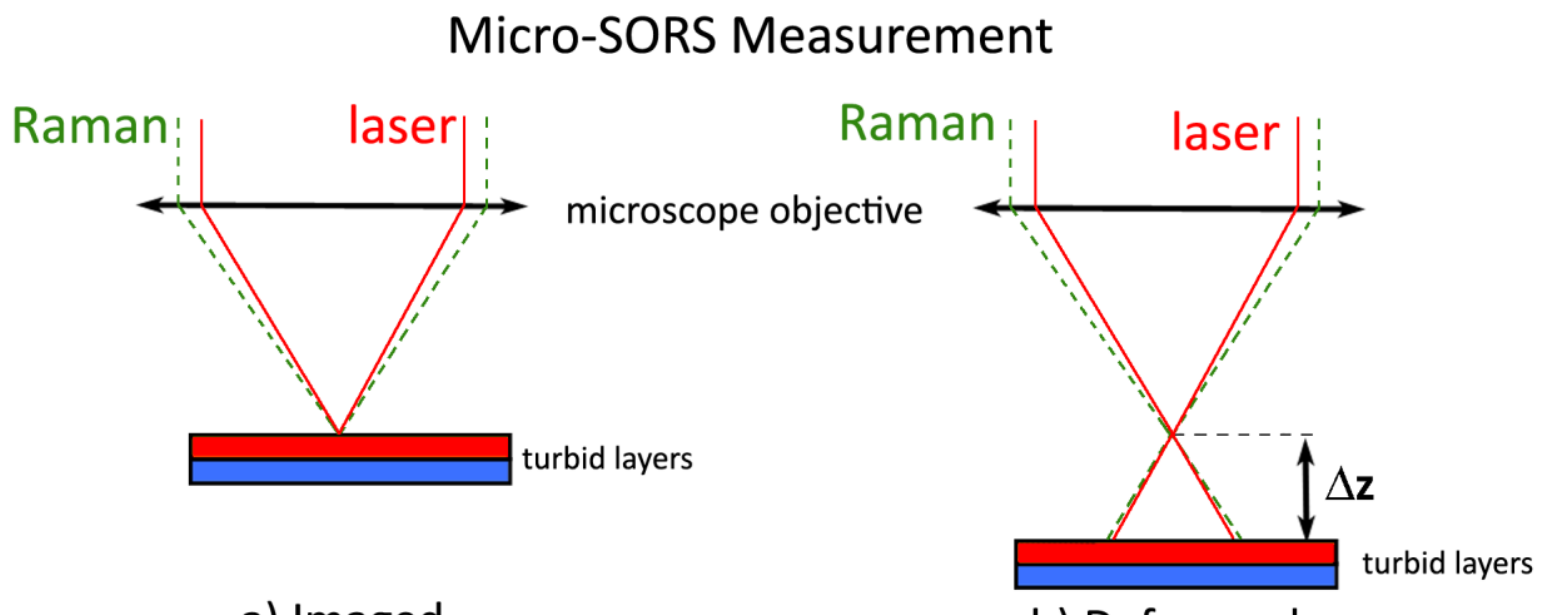

a) Imaged

b) Defocused

Figure 1: Schematic of the defocusing micro-SORS measurement consisting of acquisitions at a) 'imaged' and (b) 'defocused' positions with turbid samples.

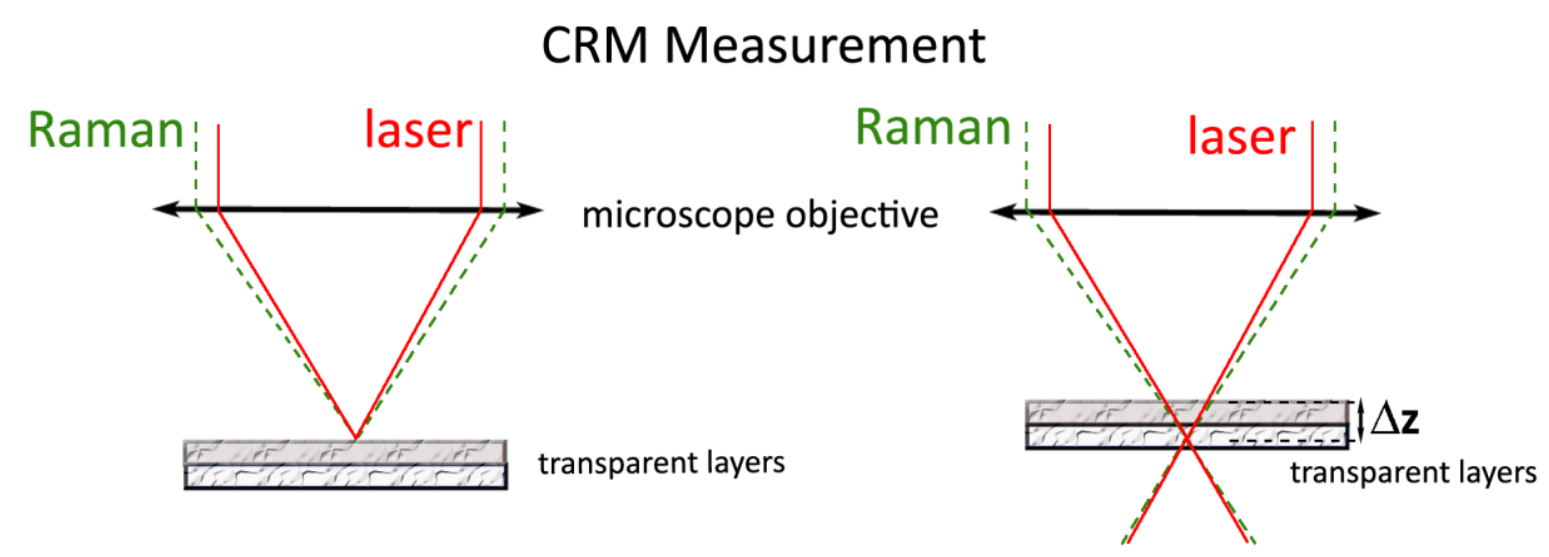

a) Surface imaged

b) Subsurface zone imaged

Figure 2: CRM z-scan (with transparent samples). 


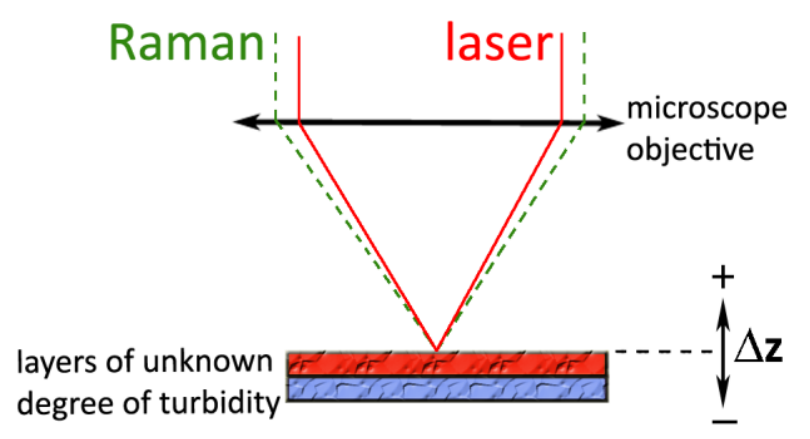

Figure 3: Proposed positive/negative z-scan to distinguish between the CRM and micro-SORS concepts.
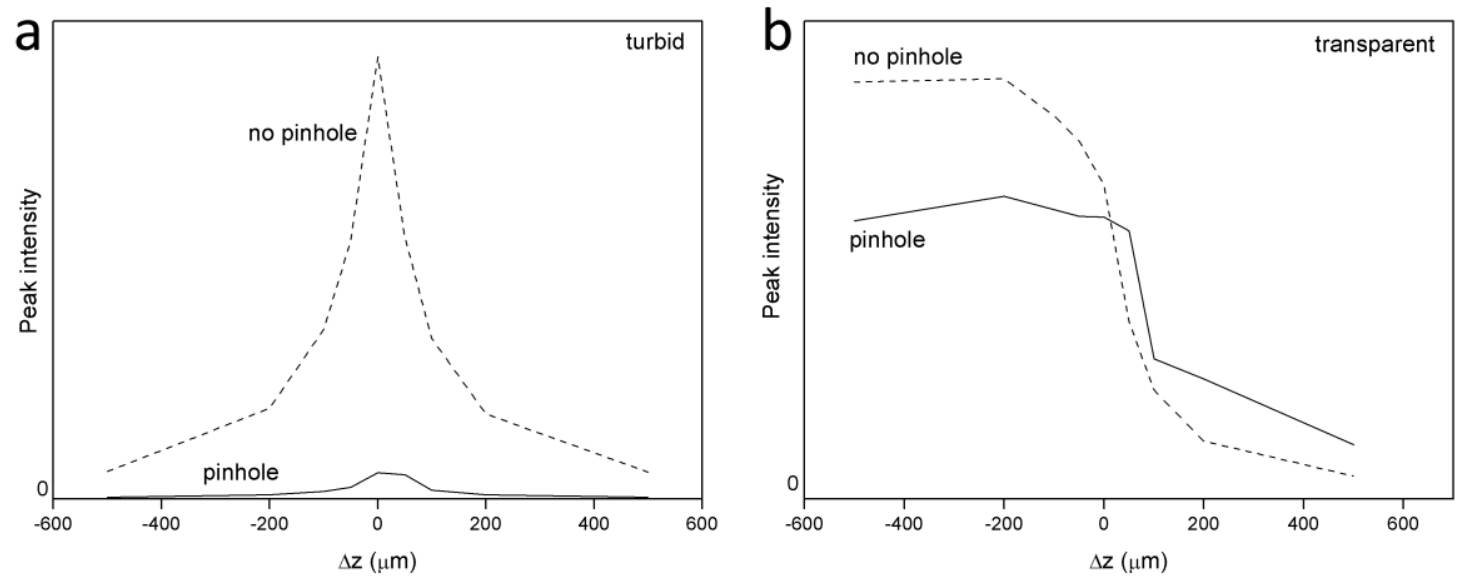

Figure 4: Dependence of Raman signal of individual layers for turbid sample (micro-SORS - a) and transparent sample (CRM - b) measured with and without confocal 'pinhole'. 

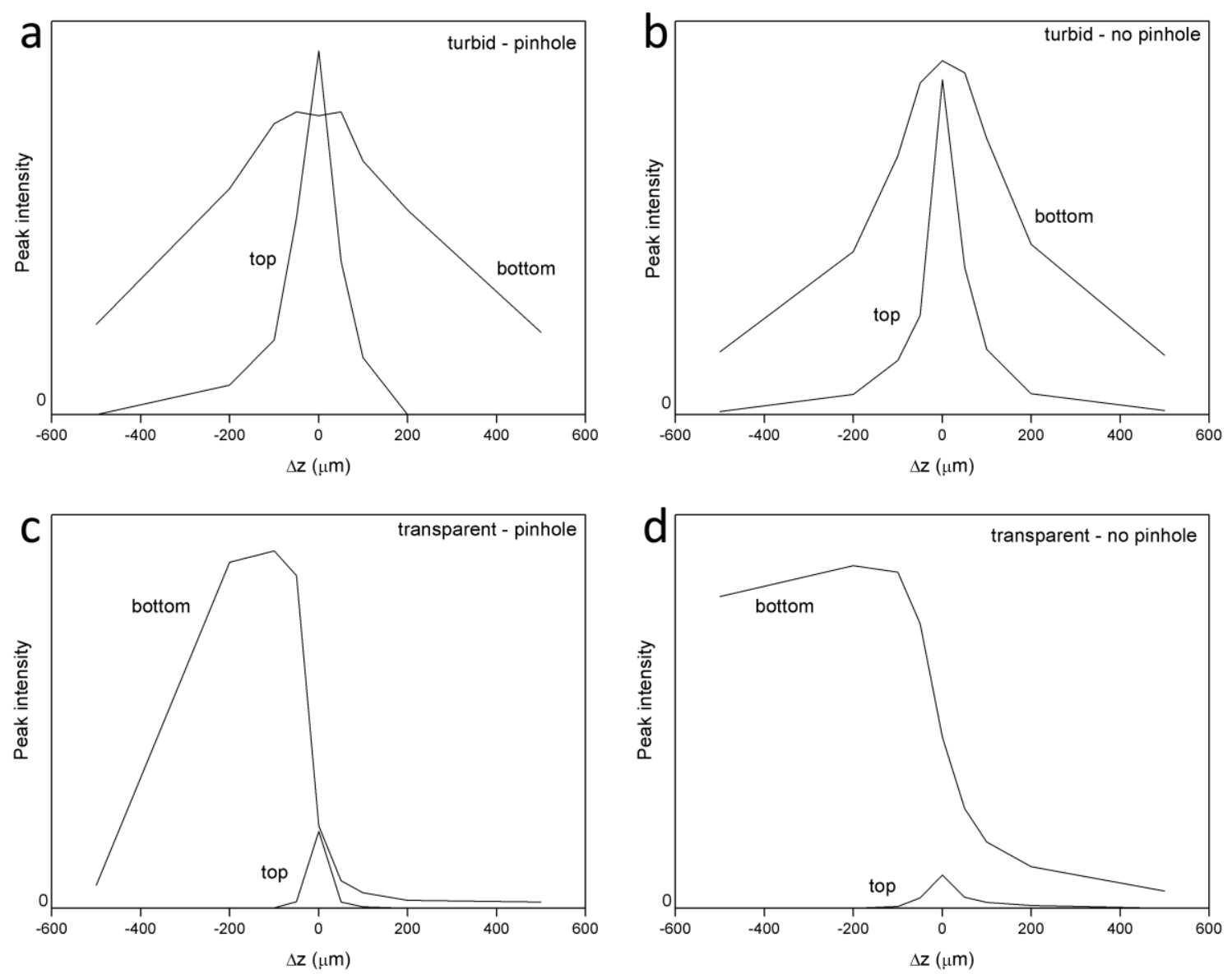

Figure 5: Dependence of Raman signal of individual layers for turbid sample (micro-SORS - a and b) and transparent sample (CRM - c and d), with and without confocal aperture ('pinhole'). 

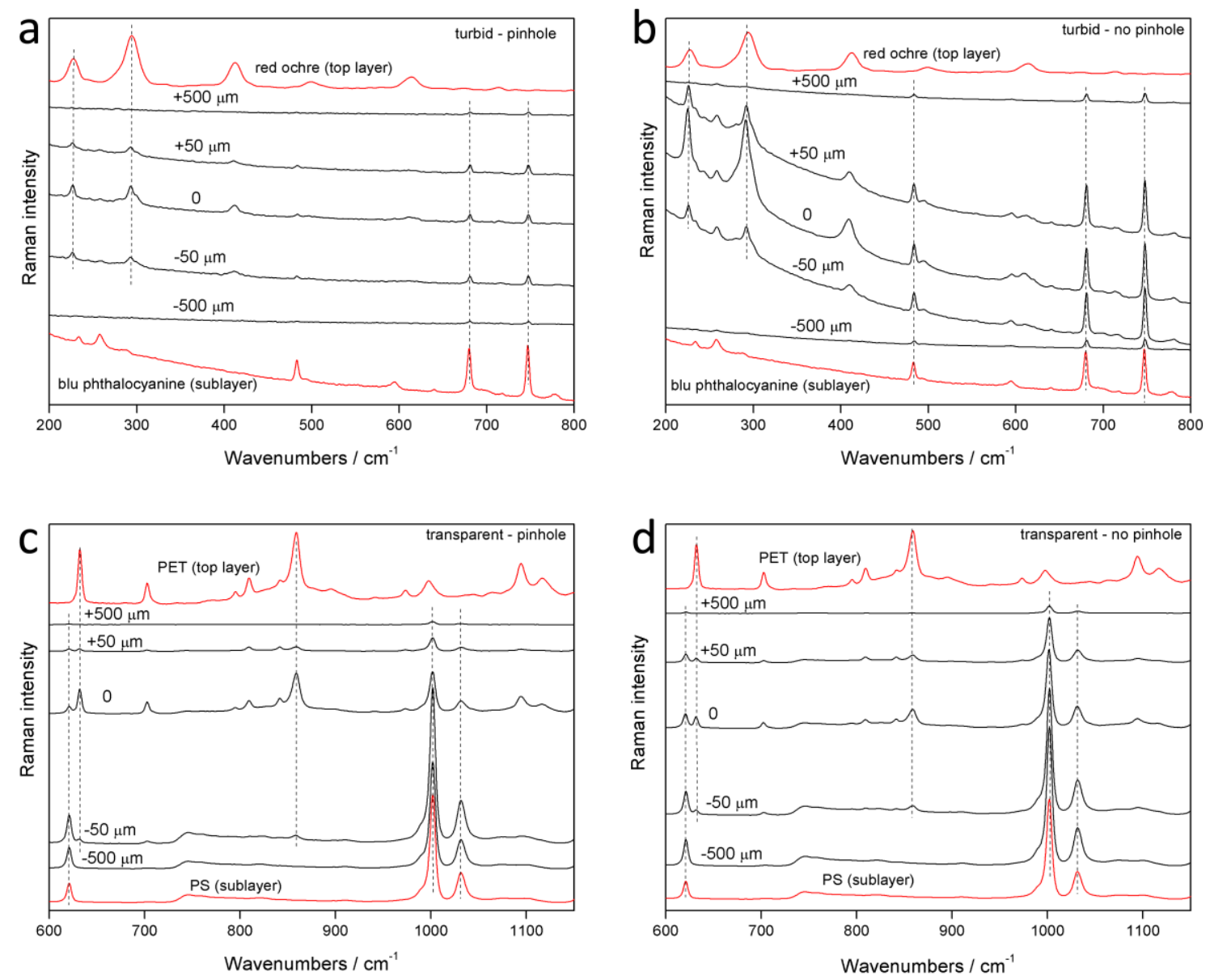

Figure 6: Example of Raman spectra from the two-layer measurements for turbid ( $a$ and $b$ ) and transparent (c and d) samples depicted in Figure 5, comparing situations with and without confocal aperture. 